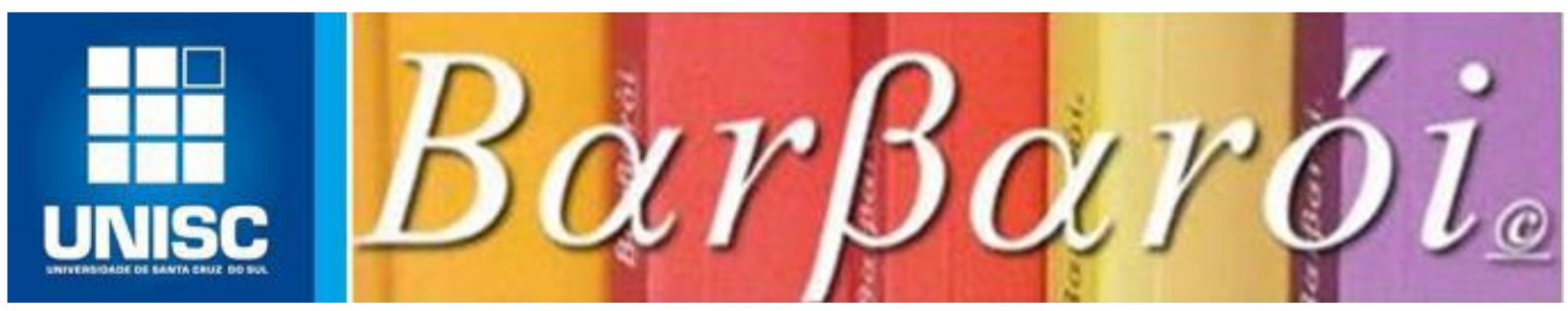

\title{
PROTAGONISMO DO USUÁRIO NA ASSISTÊNCIA EM SAÚDE MENTAL: UMA PESQUISA EM BASE DE DADOS
}

\author{
DOI: http://dx.doi.org/10.17058/barbaroi.v0i58.15125 \\ $*$ \\ Hércules Rigoni Bossato \\ Universidade Federal do Rio de Janeiro - UFRJ - Brasil \\ Virginia Faria Damasio Dutra \\ Universidade Federal do Rio de Janeiro - UFRJ - Brasil
}

\author{
Albert Lengruber de Azevedo \\ Centro Universitário Belford Roxo - UNIABEU - Brasil \\ Paula Cristina da Silva Cavalcanti \\ Associação Educacional Dom Bosco - AEDB - Brasil \\ Cristina Maria Douat Loyola \\ Universidade CEUMA-Brasil \\ Rosane Mara Pontes de Oliveira \\ Universidade Federal do Rio de Janeiro - UFRJ - Brasil
}

$*$

\section{Resumo}

Objetivo: analisar na literatura científica global o tema protagonismo do usuário no serviço de saúde mental. Método: revisão integrativa da literatura, a partir de artigos em português, inglês e francês nas bases de dados da Biblioteca Virtual em Saúde, CINAHL, SCOPUS e PubMed com palavras-chave e MESH, sem determinação temporal. Elegeram-se 21 artigos pelo fluxograma de seleção no período de agosto de 2017 a abril 2020. Resultados: o protagonismo na área da saúde mental é descrito como uma prática transformadora e humanitária pautada nas necessidades do usuário, os artigos nacionais e internacionais trouxeram concepções e ações sobre o protagonismo. Conclusão: o Modelo da Maré, as oficinas terapêuticas, o Projeto Terapêutico Singular, o Psicodrama e o teatro são ferramentas que se apresentam essenciais para o protagonismo do usuário na assistência em saúde mental, pois demarcam tecnologias e dispositivos de produção que permeiam a autonomia, a valorização e subjetividade da pessoa assistida.

Palavras-chave: Protagonismo, Assistência à Saúde Mental, Autonomia Pessoal. 


\section{Introdução}

O protagonismo nasceu na dramaturgia. O termo foi introduzido por Ésquilo, um dramaturgo da Grécia antiga e reconhecido como o pai da tragédia. A pessoa protagonista se refere aquele que possui o papel principal em uma cena, ou a primeira pessoa a falar, é mencionado também para a pessoa principal de um trabalho, obra ou um projeto (COSTA e PAULON, 2012).

No Sistema Único de Saúde (SUS) o protagonismo da pessoa assistida em um serviço de saúde mental tem por finalidade permitir que o usuário possa exercer a sua participação social no cotidiano da clínica, bem como na construção coletiva dos projetos terapêuticos (COSTA e PAULON, 2012; JORGE et. al.2015).

Entretanto, há dificuldade em mudar velhos paradigmas, a fim de proporcionar o protagonismo do usuário na terapêutica e nos serviços de saúde mental, pois mesmo com reforma psiquiátrica, a qual direciona os serviços de saúde mental ao modelo psicossocial, há alguns serviços ainda pautados no velho modelo médico tradicional (SANTIAGO e SANTOS, 2019).

Todavia, o protagonismo, segundo Costa e Paulo (2015, p.581): "requer a constituição de espaços em que os usuários possam experimentar a capacidade de pensar, sentir e decidir sobre suas vidas singulares, em meio ao coletivo que constituem e que os constitui subjetivamente."

O II Seminário Nacional de Promoção à Saúde, em Brasília, trouxe discussões sobre os dispositivos de cuidado em saúde utilizado para a ampliação das diretrizes de participação social e promotoras das políticas públicas de saúde. O seu escopo é despertar nos profissionais um olhar para as reais necessidades do usuário e produzir no seu dia a dia uma nova perspectiva sobre ele, tornando-o, assim, protagonista dos seus projetos terapêuticos e dimensionamento do cuidado em saúde (JORGE et.al. 2015; MALTA et.al. 2014).

Para Costa e Paulon (2012), o protagonismo na assistência em saúde mental acontece quando o usuário é estimulado a falar por si, em primeiro lugar, e a se reconhecer como cidadão (1). Dessa forma, ele poderá também enunciar em nome de um coletivo e permitirá ser valorizado em sua voz. Isto posto, é a partir desse pronunciamento que o usuário faz suas escolhas, definindo quais normas são essenciais para sua vida. De modo geral, é na sua participação ativa que protagonismo acontece e a autonomia da pessoa se consolida, por conseguinte, os novos rumos são dados por ele à sua vida. 
Portanto, é necessário esmaecer formas terapêuticas que permitem a valorização do protagonismo, ora presentes nos serviços da assistência em saúde mental e que se encontram há décadas sustentados em uma lógica de cuidado antimanicomial. Em meio a essas dificuldades que envolvem o processo de trabalho na área da saúde mental, há ainda uma outra questão: o saber dos profissionais de saúde (FABRI e LOYOLA, 2014).

Segundo Gruska e Dimensten (2015), o saber fazer na Reabilitação Psicossocial tece, no contexto da contemporaneidade, uma prática clínica de conduta ética, baseado em ações que promova a autonomia, principalmente daquele que se encontra em tratamento. Dentre os seus objetivos estão: aumentar as habilidades e competências do usuário, amenizar as desabilidades relacionadas ao transtorno mental ou sofrimento psíquico, e diminuir os danos gerados pela doença ao longo de sua vida.

Conforme Ribeiro e Bezerra (2015), para que a atenção psicossocial tenha êxito e traga a garantia de que o usuário seja o protagonista desse processo são primordiais as trocas sociais no intuito de proporcionar o alcance da autonomia. Destarte, de acordo com Alves et.al. (2013) na reabilitação psicossocial, o empoderamento do usuário como uma diretriz fundamental para que esse indivíduo desfrute do seu poder próprio, inerente à sua condição, a fim de realizar as suas escolhas e decisões, isto é, apropriar-se da direção de suas vidas.

Para Freitas e Amarante (2017), o desafio é romper com o que está protocolado, prescrito e direcionado para o usuário, desde os diagnósticos médicos à medicação, condutas que norteiam, ainda, o cuidado em saúde mental, e que institucionalizam cada vez mais os serviços de saúde, mesmo os substitutivos.

Nessa consideração, surge como necessidade a operacionalização de ações, nos serviços de saúde mental, que valorizem a subjetividade da pessoa e possibilitem mecanismos para dar voz ao usuário na assistência em saúde, com o propósito de despertar meios para o protagonismo, ao conceder que o usuário participe da tomada de decisão sobre a terapêutica. Ao circunscrever todo esse contexto, delineou-se para essa pesquisa a seguinte questão norteadora: de que forma são descritas as ações para o protagonismo do usuário na assistência em saúde mental? Para tanto, o estudo tem como objetivo analisar na literatura científica global o tema protagonismo do usuário na assistência em saúde mental. 


\section{Método}

Por meio de revisão integrativa da literatura, a partir de estudos publicados em bases de dados eletrônicas nacionais e internacionais, que possuíam as evidências científicas sobre o protagonismo do usuário no serviço de saúde mental.

Segundo Mendes et. al (2008) e Torraco (2016), a revisão integrativa da literatura tem por finalidade reunir e sintetizar resultados de pesquisa sobre um delimitado tema ou questão, de maneira sistemática e ordenada, com rigor científico, a fim de contribuir e aprofundar a análise do conhecimento investigado. O método da revisão integrativa pode gerar novas descobertas e perspectivas sobre o assunto pesquisado.

O estudo da revisão é realizado por meio das seguintes etapas: elaboração da pergunta norteadora, seguida pela busca biográfica, coleta de dados, análise crítica dos estudos incluídos, discussão dos resultados e considerações da revisão (MENDES, et.al 2008).

A coleta de dados desta revisão foi realizada em sete fases (Figura 1).

Figura 1 - Fluxograma das setes etapas para a coleta de dados. Rio de janeiro, RJ, 2020.

Fases Descrição da etapa

$1^{\mathrm{a}} \quad$ Acesso às bases de dados por intermédio dos periódicos CAPES.

$2^{\mathrm{a}} \quad$ Busca avançada na base no idioma português, utilizando as palavras chaves: Protagonismo AND Saúde Mental nos títulos, resumos e assuntos; Contagem dos Artigos pré-selecionados

$3^{\mathrm{a}} \quad$ Busca avançada nas bases no idioma inglês, utilizando Protagonist (MESH) AND Mental Health (MESH) nos títulos, resumos e assuntos; Contagem dos Artigos préselecionados

$4^{\mathrm{a}} \quad$ Avaliação dos artigos pré-selecionados por meio dos critérios de inclusão e exclusão do estudo

$5^{\mathrm{a}} \quad$ Avaliação dos estudos por meio da pergunta norteadora

$6^{\mathrm{a}} \quad$ Aplicação da ferramenta COREQ e categorização do nível de evidência na amostra final

$7^{\mathrm{a}} \quad$ Elaboração dos quadros finais da amostra

Fonte: elaborado pelos autores

A busca de artigos se deu a partir do acesso, em meio digital, do link periódicos CAPES no período de agosto de 2017 a abril de 2020. Esse mecanismo de busca possibilita o acesso às principais bases de dados da área da saúde. A seguinte ordem para a busca nas bases de dados, Barbarói, Santa Cruz do Sul, n. 58, p.<95-121>, jan./jun. 2021 
estabelecida de maneira aleatória, foi da seguinte forma: $1^{\circ}$ ) Biblioteca Virtual em Saúde (BVS)/Bireme; $2^{\circ}$ ) CINAHL (Cumulative Index to Nursing \& Allied Health Literature) with Full Text (EBSCO); $3^{\circ}$ ) SCOPUS; e $4^{\circ}$ ) PubMed Central (PMC).

Diferente de outras revisões integrativas que utilizam uma única plataforma de busca, este estudo elencou quatro plataformas virtuais. Elas são as principais bases de dados e bibliotecas virtuais com acervo em estudos na área da saúde e tem a finalidade de ampliar as possibilidades da busca em uma perspectiva global sobre o tema. A escolha dessas bases devese ao fato delas propiciarem um significativo acervo científico na área da assistência em saúde mental. Lembrando que apenas foram incluídos os estudos disponíveis em texto completo. Ressalta-se ainda que os estudos duplicados foram computados apenas uma vez.

Priorizou-se, inicialmente, o acesso à base de dados da Biblioteca Virtual em Saúde (BVS) (www.bireme.br). A busca avançada com as palavras-chave: protagonismo AND Saúde Mental, presente nos títulos, resumo e assunto, o boleando AND para rastreamento. Posteriormente, uma nova busca avançada foi realizada, considerando-se o idioma inglês, para o acesso às bases de dados internacionais: CINAHL; SCOPUS; e PubMed. Nessas bases, foram utilizados os descritores MESH: "Protagonist" AND "Mental Health" e o boleando AND a fim de realizar o rastreamento nas bases de dados internacionais (Quadro 1).

Quadro 1 - Estratégia de busca eletrônica nas bases de dados e bibliotecas virtuais. Rio de janeiro, RJ, Brasil 2020.

\begin{tabular}{|l|c|c|}
\hline Bases de dados e bibliotecas virtuais & $\begin{array}{l}\text { Total de artigos pré- } \\
\text { selecionados }\end{array}$ & Amostra final \\
\hline $\begin{array}{l}\text { 1. } \\
\begin{array}{l}\text { BVS (Biblioteca Virtual em Saúde) } \\
\text { BIREME. } \\
\text { Palavras chaves: Protagonismo AND } \\
\text { Saúde Mental nos títulos, resumo e } \\
\text { assunto }\end{array}\end{array}$ & 84 \\
\hline 2. CINAHL with Full Text (EBSCO) & 18 & 04 \\
\hline 3. SCOPUS (Elsevier) & 83 & 02 \\
\hline 4. PubMed Central (PMC) & 187 & 03 \\
\hline Total: & 372 & 21 \\
\hline
\end{tabular}

Fonte: elaborado pelos autores 
Cabe ressaltar que não foi delimitado um recorte temporal para a busca nas bases de dados, com a justificativa de se obter um maior número de informações possíveis sobre o tema em diversos contextos e períodos indexados.

Os critérios para a busca das fontes foram: Inclusão: textos completos nos idiomas português, inglês e francês, estudos que versem sobre o protagonismo do indivíduo que é assistido nos serviços em saúde mental. Exclusão: produções disponíveis apenas como resumos, cartas ou editoriais, teses, dissertações, monografias e livros; estudos que abordem o protagonismo dos usuários relacionados ao contexto escolar ou trabalho; protagonismo do profissional e da gestão e o protagonismo em pesquisas.

Subsequente, na etapa de seleção, os artigos foram revisados para verificação de duplicatas. Dois revisores responsabilizaram-se por analisar os títulos e/ou resumos dos artigos e remover aqueles que não estavam com textos completos. Esses mesmos revisores fizeram a leitura integral, em tela eletrônica, dos artigos encontrados, separando aqueles que se referiam à temática e aos critérios de inclusão do estudo (Quadro 1).

Ressalta-se que a seleção da amostra do estudo também é analisada com a ajuda da ferramenta Consolidated Criteria for Reporting Qualitative Research (COREQ), que segundo Tong et.al. (2007) constitui em um checklist de pontuação entre 1 e 32 itens, composto por três domínios: domínio um (Equipe de pesquisa e interferência nos dados), domínio dois (Desenho do estudo) e domínio três (Análise e achados)

O nível de evidência científica dos estudos é identificado por meio da classificação hierárquica das evidências para avaliação de pesquisas ou outra fonte de informações baseadas na categorização da Agency for Healthcare Research and Quality (AHRQ) dos Estados Unidos da América (Quadro 2). Essa categorização é avaliada por tipos de estudos tanto de abordagem quantitativa quanto qualitativa. A avaliação é realizada por sete níveis hierárquicos, sendo que o nível 1 é o maior grau e nível 7 o menor grau (MELNYK e FINCOUT-OVERHOLT, 2005; STETLER, 2001).

Quadro 2 - Nível de evidências por tipo de estudos, Rio de Janeiro, RJ, Brasil, 2020.

\begin{tabular}{|c|l|}
\hline NE* $^{*}$ & \multicolumn{1}{c|}{ TIPOS DE ESTUDOS } \\
\hline 1 & Revisões sistemáticas ou meta-análise de relevantes ensaios clínicos. \\
\hline 2 & $\begin{array}{l}\text { Evidências de, pelo menos, um ensaio clínico randomizado controlado bem } \\
\text { delineado. }\end{array}$ \\
\hline 3 & Ensaios clínicos bem delineados sem randomização. \\
\hline
\end{tabular}

Barbarói, Santa Cruz do Sul, n. 58, p.<95-121>,jan./jun. 2021 


\begin{tabular}{|c|l|}
\hline 4 & Estudos de coorte e de caso controle bem delineados. \\
\hline 5 & Revisão sistemática de estudos descritivos e qualitativos. \\
\hline 6 & Evidencias derivadas de um único estudo descritivo ou qualitativo. \\
\hline 7 & $\begin{array}{l}\text { Opinião de autoridades ou comitês de especialistas, incluindo interpretações de } \\
\text { informações não baseadas em pesquisas. }\end{array}$ \\
\hline
\end{tabular}

Fonte: Agency for Healthcare Research and Quality (AHRQ).

*NE: Nível Evidência

\section{Resultados}

Foram encontrados na amostra inicial 372 estudos nas bases de dados nacionais e internacionais. Destes, apenas 21 estudos foram separados para leitura plena e análise final, uma vez que entraram no critério de inclusão e respondiam à questão de pesquisa (Quadro 1).

Todos os artigos foram codificados (A1 a A21) para melhor identificação e relação dos achados. O quadro 3 expõe as características dos estudos segundo autores, referência, ano de publicação, país, idioma, local de busca e base de dados. A seguir, o quadro 4 caracteriza os artigos segundo a temática, objetivo e delineamento, tipo de estudo, desfecho e nível de evidência.

Quadro 3 - Caracterização dos estudos segundo código A1 a A21, autores, referência, ano de publicação, país, idioma, local de busca, base de dados. Rio de Janeiro, RJ, Brasil, 2020.

\begin{tabular}{|l|l|l|l|l|}
\hline Código & Autor (referência) & Ano & País e Idioma & $\begin{array}{l}\text { Base de dados e local } \\
\text { de busca }\end{array}$ \\
\hline A1 & Pacheco SUC, et al & 2018 & Brasil/Português & BVS/ LILACS \\
\hline A2 & Silva TA, et al & 2018 & Brasil/Português & BVS/ LILACS \\
\hline A3 & Portugal CM, et al & 2018 & Brasil/Português & BVS/ LILACS \\
\hline A4 & Kantorski LP, et al & 2017 & Brasil/Português & BVS/ LILACS \\
\hline A5 & Coelho RS, et al & 2017 & Brasil/Português & BVS/ LILACS \\
\hline A6 & Vasconcelos MGF, et al & 2016 & Brasil/Português & BVS/ LILACS \\
\hline A7 & Pitta AMF, et al & 2015 & Brasil/Português & BVS/ LILACS \\
\hline A8 & Andrade LLA e Velôso TMG & 2015 & Brasil/Português & BVS/ LILACS \\
\hline
\end{tabular}

Barbarói, Santa Cruz do Sul, n. 58, p.<95-121>,jan./jun. 2021 


\begin{tabular}{|l|l|l|l|l|}
\hline A9 & Junqueira AMG, et al & 2015 & Brasil/Português & BVS/ LILACS \\
\hline A10 & Passos E, et al & 2013 & Brasil/Português & BVS/ LILACS \\
\hline A11 & Costa DFC, Paulon SM & 2012 & Brasil/Português & BVS/ LILACS \\
\hline A12 & Torre EHG, Amarante P & 2001 & Brasil/Português & BVS/ LILACS \\
\hline A13 & Dutra VFD, et al & 2017 & Brasil/Português & CINAHL \\
\hline A14 & Ruelland I & 2015 & Francês/ Canadá & CINAHL \\
\hline A15 & Kidd J & 2010 & Inglês/Austrália & CINAHL \\
\hline A16 & Sumsion T e Lencucha R & 2007 & Inglês/ Inglaterra & CINAHL \\
\hline A17 & Gonzalez AJ, et al & 2018 & Inglês/Portugal & SCOPUS \\
\hline A18 & Pinho ES, et al & 2018 & Português/ Brasil & SCOPUS \\
\hline A19 & Barker P, Barker PB & 2010 & Inglês/ Escócia & PubMed \\
\hline A20 & Konopik DA e Cheug M & 2013 & Inglês/EUA & PubMed \\
\hline A21 & Lory Y et. al. & 2012 & Inglês/ Irã & PubMed \\
\hline
\end{tabular}

Fonte: elaborado pelos autores

Observou-se que as publicações estavam distribuídas da seguinte maneira: 12 (57\%) estudos na BVS e na base de dados LILACS, quatro (19\%) artigos na CINAHL, dois (10 \%) trabalhos na SCOPUS e três (14\%) na PubMed (Tabela 01). O idioma predominante foi o português, com $67 \%$ das publicações, seguido do inglês com $28 \%$ e do Francês com $5 \%$. Os países de origem das publicações foram: Brasil, Austrália, Irã, Portugal, Inglaterra, Escócia, EUA e Canadá. Dessa forma, $67 \%$ da amostra foi nacional e 33\% da amostra foram de estudos internacionais. A amostra corresponde a uma perspectiva global, uma vez que os artigos analisados foram publicados em diversos países bem como em distintos continentes (Quadro $3)$. 
Quadro 4 - Caracterização dos estudos, segundo código A1 a A21, temática, objetivo e delineamento, tipo de estudo, desfecho e nível de evidência. Rio de Janeiro, RJ, Brasil, 2020.

\begin{tabular}{|c|c|c|c|c|}
\hline Código & Temática & $\begin{array}{l}\text { Objetivo e delineamento/ } \\
\text { Tipo de Estudo }\end{array}$ & Desfecho & $\mathrm{NE}^{*}$ \\
\hline A1 & Empoderamento & $\begin{array}{l}\text { Demonstrar a importância de } \\
\text { protagonizar o papel ativo do } \\
\text { usuário no tratamento em } \\
\text { saúde mental/Estudo de } \\
\text { Caso }\end{array}$ & $\begin{array}{l}\text { Melhor adesão e } \\
\text { aceitação do usuário por } \\
\text { meio da valorização da } \\
\text { sua voz nos espaços } \\
\text { terapêuticos. }\end{array}$ & 6 \\
\hline A2 & $\begin{array}{l}\text { Ações } \\
\text { psicossociais }\end{array}$ & $\begin{array}{l}\text { Apresentar as ações } \\
\text { desenvolvidas em Centro de } \\
\text { Atenção Psicossocial/Estudo } \\
\text { retrospectivo/ descritivo- } \\
\text { exploratório }\end{array}$ & $\begin{array}{l}\text { Ações do Centro de } \\
\text { Atenção Psicossocial } \\
\text { (CAPS) fortalecem o } \\
\text { protagonismo de } \\
\text { usuários }\end{array}$ & 6 \\
\hline A3 & $\begin{array}{l}\text { Espaços para o } \\
\text { protagonismo }\end{array}$ & $\begin{array}{l}\text { Discutir os efeitos } \\
\text { transformadores dos } \\
\text { contextos militantes e } \\
\text { artísticos no cotidiano dos } \\
\text { usuários de saúde } \\
\text { mental/Estudo etnográfico }\end{array}$ & $\begin{array}{l}\text { A importância de } \\
\text { espaços abertos para o } \\
\text { protagonismo dos } \\
\text { sujeitos e para uma } \\
\text { reforma psiquiátrica } \\
\text { pensada enquanto } \\
\text { processo social } \\
\text { complexo. }\end{array}$ & 6 \\
\hline A4 & $\begin{array}{l}\text { Oficina } \\
\text { terapêutica }\end{array}$ & $\begin{array}{l}\text { Discutir as estratégias } \\
\text { utilizadas por diferentes } \\
\text { grupos de pessoas que } \\
\text { ouvem vozes/Estudo } \\
\text { etnográfico }\end{array}$ & $\begin{array}{l}\text { A oficina compartilha as } \\
\text { estratégias de } \\
\text { enfretamento estimulam } \\
\text { o protagonismo do } \\
\text { usuário }\end{array}$ & 6 \\
\hline A5 & $\begin{array}{l}\text { Oficina } \\
\text { terapêutica }\end{array}$ & $\begin{array}{l}\text { Refletir sobre o papel da } \\
\text { família na rede intersetorial } \\
\text { de saúde mental/Relato de } \\
\text { experiência }\end{array}$ & $\begin{array}{l}\text { A oficina entre o usuário } \\
\text { e o familiar são } \\
\text { estratégias para resolver } \\
\text { problemas, o que denota } \\
\text { posturas mais } \\
\text { participativas e críticas. }\end{array}$ & 6 \\
\hline
\end{tabular}




\begin{tabular}{|c|c|c|c|c|}
\hline A6 & $\begin{array}{l}\text { Projeto } \\
\text { Terapêutico }\end{array}$ & $\begin{array}{l}\text { Compreender o cuidado } \\
\text { psicossocial/Hermenêutica }\end{array}$ & $\begin{array}{l}\text { Escassez de discussões } \\
\text { que considerassem o } \\
\text { protagonismo dos } \\
\text { usuários e da família na } \\
\text { construção do projeto } \\
\text { terapêutico }\end{array}$ & 6 \\
\hline A7 & $\begin{array}{l}\text { Direitos } \\
\text { humanos }\end{array}$ & $\begin{array}{l}\text { Avaliar as práticas dos } \\
\text { Centros de Atenção } \\
\text { Psicossocial no respeito aos } \\
\text { direitos humanos dos } \\
\text { usuários/Etnoepidemiológico }\end{array}$ & $\begin{array}{l}\text { Os Centros de Atenção } \\
\text { Psicossocial influenciam } \\
\text { positivamente na vida } \\
\text { dos usuários, ao } \\
\text { estimular-lhes a } \\
\text { autonomia, a } \\
\text { corresponsabilização no } \\
\text { cuidado e o } \\
\text { protagonismo. }\end{array}$ & 6 \\
\hline A8 & $\begin{array}{l}\text { Terapia com } \\
\text { teatro }\end{array}$ & $\begin{array}{l}\text { Relatar uma experiência de } \\
\text { estágio desenvolvida em um } \\
\text { Centro de Atenção } \\
\text { Psicossocial/Pesquisa } \\
\text { participante }\end{array}$ & $\begin{array}{l}\text { O teatro do oprimido } \\
\text { promoveu saúde e } \\
\text { estimulou a autonomia e } \\
\text { o protagonismo social } \\
\text { dos usuários. }\end{array}$ & 6 \\
\hline A9 & $\begin{array}{l}\text { Assembleia dos } \\
\text { usuários }\end{array}$ & $\begin{array}{l}\text { Investigar a Assembleia } \\
\text { como uma modalidade de } \\
\text { grupo que também pode ser } \\
\text { terapêutica/Estudo } \\
\text { exploratório em diário de } \\
\text { campo e pesquisa } \\
\text { documental }\end{array}$ & $\begin{array}{l}\text { Assembleia como um } \\
\text { espaço que legitima a } \\
\text { inserção dos usuários } \\
\text { nas decisões } \\
\text { institucionais e a maior } \\
\text { participação em seu } \\
\text { tratamento. }\end{array}$ & 6 \\
\hline A10 & $\begin{array}{l}\text { Gestão } \\
\text { autônoma da } \\
\text { medicação }\end{array}$ & $\begin{array}{l}\text { Elaborar do Guia Brasileiro } \\
\text { da Gestão Autônoma da } \\
\text { Medicação (Guia GAM-BR) } \\
\text { / Estudo Multicêntrico } \\
\text { Exploratório }\end{array}$ & $\begin{array}{l}\text { A estratégia GAM } \\
\text { assumiu o desafio de } \\
\text { propor-se como prática } \\
\text { congestiva, } \\
\text { compatibilizando } \\
\text { exercício da autonomia, }\end{array}$ & 6 \\
\hline
\end{tabular}

Barbarói, Santa Cruz do Sul, n. 58, p.<95-121>,jan./jun. 2021 


\begin{tabular}{|c|c|c|c|c|}
\hline & & & $\begin{array}{l}\text { direito e protagonismo } \\
\text { do usuário. }\end{array}$ & \\
\hline A11 & $\begin{array}{l}\text { Protagonismo e } \\
\text { participação } \\
\text { social }\end{array}$ & $\begin{array}{l}\text { Analisar o exercício de } \\
\text { protagonismo e participação } \\
\text { social de usuários em saúde } \\
\text { mental/Cartografia }\end{array}$ & $\begin{array}{l}\text { O conceito de coletivo } \\
\text { indicou a potência de um } \\
\text { novo arranjo } \\
\text { participativo, capaz de } \\
\text { dialogar com as noções } \\
\text { de autonomia e } \\
\text { protagonismo. }\end{array}$ & 6 \\
\hline A12 & $\begin{array}{l}\text { Protagonismo e } \\
\text { subjetividades }\end{array}$ & $\begin{array}{l}\text { Refletir sobre as origens e as } \\
\text { bases históricas e conceituais } \\
\text { da produção de subjetividade } \\
\text { do sujeito considerado } \\
\text { louco/Estudo reflexão por } \\
\text { meio genealogia da } \\
\text { subjetividade }\end{array}$ & $\begin{array}{l}\text { As práticas atuais no } \\
\text { campo da saúde mental } \\
\text { que têm como proposta a } \\
\text { construção coletiva do } \\
\text { sujeito da loucura, não } \\
\text { mais como sujeito } \\
\text { alienado, mas como } \\
\text { protagonista, isto é, de } \\
\text { uma nova relação social } \\
\text { com a loucura }\end{array}$ & 6 \\
\hline A13 & Autonomia & $\begin{array}{l}\text { Identificar e analisar as } \\
\text { práticas da Enfermagem } \\
\text { Psiquiátrica na mediação da } \\
\text { autonomia de sujeitos sociais } \\
\text { comprometidos } \\
\text { pela sua história } \\
\text { psiquiátrica/Pesquisa } \\
\text { Convergente Assistencial } \\
\text { (PCA) }\end{array}$ & $\begin{array}{l}\text { Os enfermeiros apostam } \\
\text { na autonomia e na } \\
\text { liberdade como } \\
\text { fundamento do cuidado } \\
\text { que poderão ser } \\
\text { estimuladas, mediadas } \\
\text { ou negociadas. }\end{array}$ & 6 \\
\hline A14 & Assembleias & $\begin{array}{l}\text { Descrever como as trocas } \\
\text { criadas dentro e } \\
\text { fora da assembleia dos } \\
\text { usuários repercutem em sua }\end{array}$ & $\begin{array}{l}\text { As assembleias podem } \\
\text { compartilhar espaços } \\
\text { sociais para os afetos e } \\
\text { permiti um lugar para os } \\
\text { usuários possam criticar }\end{array}$ & 6 \\
\hline
\end{tabular}

Barbarói, Santa Cruz do Sul, n. 58, p.<95-121>,jan./jun. 2021 


\begin{tabular}{|c|c|c|c|c|}
\hline & & $\begin{array}{l}\text { participação social/Estudo de } \\
\text { caso }\end{array}$ & $\begin{array}{l}\text { livremente a assistência } \\
\text { recebida. }\end{array}$ & \\
\hline A15 & $\begin{array}{l}\text { Modelo da } \\
\text { Maré }\end{array}$ & $\begin{array}{l}\text { Explorar a natureza } \\
\text { individual dos limites } \\
\text { culturais e descobrir } \\
\text { mensagens ocultas que } \\
\text { influenciam nossos esforços } \\
\text { para construir conexões } \\
\text { entre culturas e etnias em } \\
\text { ambientes de saúde } \\
\text { mental/Estudo exploratório } \\
\text { por meio do modelo da } \\
\text { maré. }\end{array}$ & $\begin{array}{l}\text { O potencial do modelo } \\
\text { de maré fornece um } \\
\text { veículo para uma } \\
\text { compreensão cultural na } \\
\text { assistência em saúde. }\end{array}$ & 6 \\
\hline A16 & $\begin{array}{l}\text { Cuidado } \\
\text { centrado na } \\
\text { pessoa }\end{array}$ & $\begin{array}{l}\text { Realizar a aplicação de uma } \\
\text { prática centrada no } \\
\text { cliente/Estudo exploratório }\end{array}$ & $\begin{array}{l}\text { Os terapeutas usaram } \\
\text { atitudes e ações para } \\
\text { facilitar o processo } \\
\text { centrado no cliente e os } \\
\text { clientes trouxeram } \\
\text { pontos fortes para esse } \\
\text { relacionamento. }\end{array}$ & 6 \\
\hline A17 & Psicodrama & $\begin{array}{l}\text { Avaliar o psicodrama como } \\
\text { dispositivo } \\
\text { terapêutico/Estudo de caso }\end{array}$ & $\begin{array}{l}\text { Os participantes } \\
\text { relataram melhora em } \\
\text { seus objetivos } \\
\text { terapêuticos pessoais, } \\
\text { diminuição dos sintomas } \\
\text { e problemas de vida, e } \\
\text { alguns mostraram um } \\
\text { aumento acentuado nos } \\
\text { níveis de } \\
\text { espontaneidade. }\end{array}$ & 6 \\
\hline
\end{tabular}




\begin{tabular}{|c|c|c|c|c|}
\hline A18 & $\begin{array}{l}\text { Processo de } \\
\text { trabalho no } \\
\text { CAPS }\end{array}$ & $\begin{array}{l}\text { Mostrar como os processos } \\
\text { de trabalho estão sendo } \\
\text { desenvolvidos pelos } \\
\text { profissionais do CAPS e as } \\
\text { repercussões para os } \\
\text { usuários do serviço/ } \\
\text { Revisão Integrativa }\end{array}$ & $\begin{array}{l}\text { Projeto Terapêutico } \\
\text { Singular (PTS) e o } \\
\text { território com } \\
\text { componentes necessários } \\
\text { para a proposta de } \\
\text { desinstitucionalização }\end{array}$ & 6 \\
\hline A19 & $\begin{array}{l}\text { Modelo da } \\
\text { Maré }\end{array}$ & $\begin{array}{l}\text { Descrever a aplicação dos } \\
\text { vários processos de } \\
\text { tratamento de no modelo da } \\
\text { maré/Revisão Integrativa }\end{array}$ & $\begin{array}{l}\text { O Modelo de Maré pode } \\
\text { ser utilizado na } \\
\text { terapêutica em saúde } \\
\text { mental. Ele é um modelo } \\
\text { centrados na pessoa }\end{array}$ & 6 \\
\hline A20 & Psicodrama & $\begin{array}{l}\text { Examinar os resultados de } \\
\text { um tratamento de } \\
\text { psicodrama em um programa } \\
\text { de hospitalização/Estudo } \\
\text { caso }\end{array}$ & $\begin{array}{l}\text { A importância do } \\
\text { esforço combinado do } \\
\text { terapeuta e do paciente } \\
\text { fazendo do psicodrama } \\
\text { uma ponte para o } \\
\text { tratamento posterior }\end{array}$ & 6 \\
\hline A21 & $\begin{array}{l}\text { Terapia com } \\
\text { teatro }\end{array}$ & $\begin{array}{l}\text { Descrever a técnica da } \\
\text { terapia com teatro e sua } \\
\text { aplicabilidade/ } \\
\text { Estudo de reflexão }\end{array}$ & $\begin{array}{l}\text { A terapia com teatro } \\
\text { quando atinge o nível de } \\
\text { criatividade e } \\
\text { espontaneidade } \\
\text { (improvisação) facilitará } \\
\text { para o paciente expressar } \\
\text { a emoção }\end{array}$ & 6 \\
\hline
\end{tabular}

*NE: Nível de Evidência

Todos os estudos apresentam um nível de evidência seis. Portanto, a amostra final corresponde as evidências derivadas de um único estudo descritivo ou qualitativo. Com relação aos tipos de estudo: quatro (19\%) são estudos exploratórios; quatro (19\%) são estudos de casos; dois $(9,5 \%)$ estudos etnográficos; dois $(9,5 \%)$ estudos de reflexão; dois $(9,5 \%)$ estudos de revisão integrativa. Os demais são: um (4,7\%) estudo retrospectivo/descritivo-exploratório; um $(4,7 \%)$ estudo de pesquisa participante; um $(4,7 \%)$ relato de experiência; um $(4,7 \%)$ estudo Barbarói, Santa Cruz do Sul, n. 58, p.<95-121>,jan./jun. 2021 
hermenêutico; um (4,7\%) estudo etnoepidemiológico; um (4,7\%) cartografia; um (4,7\%) estudo convergente assistencial. Destarte, grande parte dos artigos utilizam a metodologia dos estudos exploratórios e o estudo de caso em pesquisas com abordagem qualitativa.

Em relação as temáticas: um estudo (A1) acena sobre o empoderamento dos usuários nos serviços de saúde mental, um estudo (A11) aborda as concepções sobre o protagonismo e participação social e um estudo (A12) analisa o protagonismo e subjetividade de forma reflexiva e conceitual. A autonomia é tratada em um estudo (A13) como um fenômeno que pode ser mediado pela enfermagem psiquiátrica na assistência em saúde mental.

Cabe destacar que, ainda em relação à temática, um estudo (A2) trouxe o Centros de Atenção Psicossocial (CAPS) como espaços que podem favorecer o protagonismo do usuário e um estudo (A18) abrangeu o processo de trabalho no CAPS. Destaca-se, um estudo (A7) com a temática os direitos humanos e a avaliação do CAPS.

Em relação ao tema gestão autônoma da medicação foi referido em um estudo (A10) e dois estudos (A9 e A14) versaram sobre a assembleia dos usuários como um espaço para os mesmos exercerem o protagonismo. O tema cuidado centrado na pessoa é abordado em um estudo (A16) e a temática Modelo da Maré esteve presente em dois estudos (A15 e A19).

Pontua-se que o tema oficina terapêutica foram discutidos em dois estudos (A4 e A5). Já o tema projeto terapêutico é descrito em um estudo (A6). Quanto a temática, espaços para o protagonismo é explorada em um estudo (A3) como uma forma de arte. Em relação ao tema, psicodrama é proposto em dois estudos (A17 e A20) e outros dois estudos (A21 e A8) coloca a terapia com teatro como ferramentas terapêuticas para o protagonismo da pessoa na assistência em saúde mental.

Ressalta-se que a abordagem acerca do protagonismo do usuário na assistência em saúde mental se deu de maneira plural em distintos países. Em síntese, o fenômeno protagonismo traz uma dimensão da tríade "ética-política-clínica" relacionando os aspectos conceituais e de construção prática, além das possibilidades terapêuticas da reabilitação psicossocial tanto na atenção primária quanto nos serviços especializados, num cenário com tendência à interdisciplinaridade e galgados no movimento da reforma psiquiátrica mundial.

Há uma considerável gama de estudos internacionais que versam sobre as abordagens terapêuticas específicas, como psicodrama, teatro livre e a adoção do modelo da maré, como dispositivos terapêuticos que repercutem em ações para o protagonismo do usuário na assistência em saúde mental. Todos eles têm o intuito de amenizar os sofrimentos psíquicos e 
exposição para o alívio dos sintomas por meio da expressão de sentimentos da pessoa que está sendo assistida, bem como a sua valorização da subjetividade.

\section{Discussão}

Embora a realização de estudos acerca da temática protagonismo do usuário não seja uma questão recente na área da saúde mental, o conhecimento produzido sobre o assunto esteve atrelado, durante décadas, à perspectiva de gestores em saúde e dos profissionais de saúde sobre o cuidado ao usuário com transtorno mental e/ou sofrimento psíquico. Conhecimento esse, por vezes, ancorado em ações de cunho apenas educativo.

Contudo, com o objetivo de promover o protagonismo do usuário na área da saúde mental a literatura global, nas últimas décadas, nos últimos cinco anos e concentração de publicações sobre o tema nos últimos dois anos trouxe as implicações sobre as abordagens terapêuticas e ferramentas de cuidado em saúde que favorecem a autonomia e o protagonismo do usuário na assistência.

O estudo analisa os temas relacionados ao protagonismo do usuário no serviço de saúde mental. Evidenciados a seguir por: Concepções e conceitos do protagonismo; o modelo da Maré e a enfermagem; as oficinas terapêuticas; o Projeto Terapêutico Singular (PTS); o psicodrama e o teatro. Essas temáticas estão relacionadas as formas terapêuticas que favorecem o protagonismo da pessoa assistida em um serviço de saúde.

\section{Concepções ligadas ao Protagonismo dos usuários na assistência em saúde mental}

A conduta clínica da reabilitação psicossocial, ao longo dos anos, vem recebendo uma maior atenção dos profissionais de saúde, por tratar-se de uma iniciativa que favorece a incorporação de novos valores e dispositivos de acompanhamento e tratamento dos usuários (24). Dentre essas práticas, está o protagonismo do usuário, que, de modo geral, apresenta-se como uma ferramenta inerente ao processo de cuidar (COSTA e PAULON, 2012)

Cabe ressaltar que, no Brasil, o reconhecimento das práticas do Centro de Atenção Psicossocial (CAPS) demonstra que, além de ser um serviço assistencial em saúde, é também um dispositivo contemporâneo de cuidado e influenciador dos estímulos positivos para a construção da autonomia e protagonismo do usuário. Esse é um espaço para a corresponsabilização do cuidado e provedor do protagonismo sociocultural e político nas suas respectivas comunidades (TORRE e AMARANTE, 2001). 
Nesse segmento, segundo Silva et.al (2018) as ações no CAPS devem promover mecanismos para fortalecer o protagonismo das pessoas assistidas. Nesse contexto, cabe ressaltar que os enfermeiros do CAPS apostam na autonomia e na liberdade como fundamento do cuidado. De forma que a autonomia para o cuidado em saúde pode ser estimulada, mediada ou negociada (DUTRA et.al 2017).

O paradigma psicossocial traz a uma nova concepção da sociedade em relação à loucura, onde o próprio usuário, antes visto como alienado, é considerado como o maior protagonista dessa construção coletiva e clínica. Esse fato, além demarcar um campo de produção de subjetividades, pela centralidade no usuário, pode tornar efetiva toda e qualquer terapêutica proposta ${ }^{(24) .}$ Nessa circunstância, os CAPS influenciam positivamente na vida das pessoas quando estimulam a autonomia, a corresponsabilização do cuidado e o protagonismo (PITTA et.al 2015)

No que se refere às dificuldades encontradas pelos profissionais de saúde, em uma prática de cuidados, pautada no protagonismo do usuário, conforme Costa e Paulon (2012), a participação social no SUS é um desafio atual, mesmo com tantos avanços na área da saúde mental, e um dos maiores dilemas para o movimento da reforma psiquiátrica brasileira .

Contudo, segundo Junqueira et.al (2015) um exemplo de cenário possível para superar as barreiras para que a pessoa exerça o seu protagonismo, é a possibilidade das assembleias dos usuários como um dispositivo de organização do serviço e da própria clínica. As assembleias são espaços que legitimam a inserção dos usuários nas decisões institucionais e a maior participação em seu tratamento no intuito de resgatar a autonomia e o protagonismo da pessoa assistida em um serviço de saúde. Dessa forma, de acordo com Ruelland (2015), as assembleias possibilitam que as pessoas compartilhem espaços sociais para despertar os afetos e ter um lugar onde eles possam criticar livremente a assistência recebida.

Nessa perspectiva, o protagonismo do usuário está ligado a participação social. Portanto, no Brasil é uma das diretrizes do SUS que visa o envolvimento da pessoa assistida nos espaços promotores de saúde para proporcionar resolutividade das necessidades em saúde. Na atenção primária brasileira, a iniciativa de dar voz ao usuário e de colocá-lo no centro da atenção demarca uma interface do protagonismo, que pode ser mediado tanto pela corresponsabilização do usuário, quanto pela responsabilização do serviço de saúde (TORRE e AMARANTE, 2001).

Conforme Passos el.al (2013) aborda um outro dispositivo específico que proporciona a pessoa exercer o seu protagonismo na assistência em saúde mental é a gestão autônoma da 
medicação (GAM). A estratégia GAM é uma prática congestiva que compartilha o exercício da autonomia e o protagonismo do usuário

Dessa forma, o protagonismo cria uma agenda nos serviços de saúde pautados pela diretriz da desinstitucionalização. A ideia é criar espaços e possibilidades que deem voz aos usuários em tratamento ou no acompanhamento da assistência em saúde mental. Para tanto, tornar-se necessário o aperfeiçoamento da terapêutica pautada em uma ética assistencial que superem as barreiras da marginalização e exclusão social.

\section{O Modelo da Maré e a Enfermagem}

O modelo da teoria da maré (Tidal Model) é construído a partir de uma prática filosófica, pautada no relacionamento terapêutico e na compreensão do usuário, por meio dos seus modos de vivenciar as crises. Esta é uma ferramenta essencial para superar as barreias de comunicação na terapêutica entre enfermeiros e os usuários dos serviços em um mundo globalizado (KIDD, 2010).

Segundo Barker e Barker (2010) a base do modelo da maré, encontra-se sustentada na teoria do Caos, no fluxo constante, nas marés que vão e vêm e mostram padrões não repetitivos, embora permaneçam dentro dos parâmetros definidos.

No estudo publicado na Escócia (A31), o modelo da Maré (Tidal Model) é apresentado como um recuso dos serviços de saúde mental que oferece uma ênfase nas ações terapêuticas, favorecendo, a partir dos planos de cuidados, a centralidade do usuário e seu protagonismo (BARKER e BARKER, 2010).

Nesse modelo clínico foi criado por enfermeiros, é uma teoria de enfermagem de médio alcance. Para sua adoção é essencial estabelecer formas de relacionamento e comunicação terapêuticos em meio às diversidades e complexidades que envolvem o cuidado em saúde mental. Portanto, o modelo da maré traz uma valorização da subjetividade do usuário e suas trocas sociais e, dessa forma, também se aproxima da Reabilitação Psicossocial e possibilita a pessoa assistida exercer o seu protagonismo no processo de cuidado.

Cabe ressaltar que o modelo da Maré é uma ferramenta terapêutica que abrange uma visão de mundo específica e a adoção de tal modelo ajuda a enfermeira e/ou profissional de saúde a começar a entender o que é a saúde mental e o que ela significa para uma determinada pessoa. Dessa maneira, é possível ajudar o indivíduo a definir e a começar a complexa e exigente jornada de recuperação, tratamento e promoção do seu protagonismo. A participação 
social do usuário, bem como o seu empoderamento são fenômenos para uma terapêutica que possibilita o caber na vida da pessoa assistida em um serviço de saúde mental.

Para tanto, conforme Sumision e Lencucha (2007) há uma potente e considerável discussão sobre o cuidado focado na centralidade do usuário. Aborda-se que a centralidade entre usuário e profissional de saúde é um fator capaz de desencadear o protagonismo, embora a família e outras pessoas também sejam autores-chave para promover o cuidado em saúde.

\section{Oficinas Terapêuticas}

As oficinas terapêuticas em saúde mental, na contemporaneidade, trazem a ideia de singularidade do sujeito, da cidadania e da possibilidade de reinserção social. É um espaço de trocas sociais onde o usuário do serviço pode aprender e aperfeiçoar uma arte, desenvolver mecanismo de trabalho e renda, demostrar habilidades de um grupo, expressar sua cultura e lazer e possibilitar a terapêutica na reabilitação psicossocial (FARIAS et.al. 2016).

Destaca-se um estudo brasileiro sobre a utilização de uma oficina terapêutica para promover o protagonismo do usuário. Esse estudo aborda as intervenções psicossociais por meio de um grupo de usuários do Centro de Atenção Psicossocial. Essas intervenções no grupo de acordo com Coelho et.al (2017,p.489): “tinham o objetivo promover o protagonismo dos usuários e sua corresponsabilização nos processos familiares, por meio de oficinas semanais sobre temas relacionados à família".

Um outro estudo discute a criação de grupo de ouvidores de vozes, por intermédio de uma oficina, a fim de realizar trocas sociais e experiências e estratégias de enfrentamento. Tal espaço estimula o protagonismo social do usuário, uma vez que a oficina tem objetivo de valorizar a experiência da pessoa em vivenciar um delírio (PORTUGAL, et.al 2018).

Portanto, o protagonismo da pessoa em um serviço de saúde mental pode ser trabalhado por meio de oficinas terapêuticas, nas quais a valorização da experiência do indivíduo e do grupo são espaços de construções coletivas para enfrentamento do problema e superação de suas barreiras. Além de favorecer a corresponsabilização e diálogos sobre questões relacionadas ao cuidado em saúde mental.

\section{O Projeto Terapêutico Singular (PTS)}

Segundo, Pinho et.al (2018) o projeto terapêutico singular (PTS) é uma ferramenta de construção interdisciplinar, por meio de plano de ação compartilhado, que visa a integralidade do cuidado. Ele é um dispositivo essencial para o processo de trabalho da equipe de saúde na 
prestação de uma assistência singularizada na pessoa. Tal dispositivo é um projeto terapêutico para o trabalho focado no território e territorialidades do usuário do serviço.

Cabe esclarecer, conforme o Ministério da Saúde (2014), que o PTS é um instrumento que permite a sistematização do cuidado construído entre o usuário do serviço e a equipe de saúde multiprofissional, que deve considerar a singularidade do sujeito e a complexidade de cada caso. Há uma participação conjunta com a família desse sujeito e um olhar sobre as potencialidades do território que a pessoa se encontra

Destaca-se que o empoderamento do usuário no CAPS na construção e avaliação dos seus projetos de terapêuticos é um mecanismo essencial para conferir a adesão do usuário e sua aceitação na evolução do processo de cuidado (PACHECO, et.al 2018).

A participação em grupo tem efeitos para o processo de protagonismo da pessoa, uma que vez que melhora adesão ao serviço e pode contribuir para o indivíduo assistido lidar com as suas questões de vida cotidiana.

Contudo, Vasconcelos et. al. (2016) aborda que há dilemas expressados pelos profissionais de saúde no Centro de Atenção Psicossocial (CAPS) em suas dimensões epistemológicas e técnico assistenciais, referentes às ações dos profissionais de saúde junto ao PTS. Este, por sua vez, passou a ter uma formatação mais fragmentada e configurada na especialidade do que uma configuração mais dinâmica e interdisciplinar.

Dessa maneira, ainda há necessidade de estudos que versam sobre o protagonismo dos usuários e familiares na construção dos Projetos Terapêuticos Singulares e nas ações terapêuticas em saúde nos serviços de saúde mental.

\section{Psicodrama e teatro}

Inspirado no teatro, o Psicodrama propõe o desempenho de papéis pela dramatização como método de desenvolvimento e análise de um fenômeno. Drama significa ação ou realização. Destarte, no Psicodrama há o desempenho livre de papéis e os seus vínculos, ampliando a espontaneidade e a criatividade, para propiciar uma abordagem psicoterápica (SANTOS e BITENCOURT, 2020).

Em relação aos estudos sobre esse assunto, como os realizados nos EUA, Escócia e Irã, grande parte deles versam sobre o reconhecimento do protagonismo do usuário, colocando-o como parte integrante da terapêutica em saúde por intermédio do psicodrama e o teatro.

Essas ações da arte como expressão terapêutica são construídas em conjunto com o usuário, no intuito de favorecer o seu protagonismo no processo de cuidado e compreensão do 
seu sofrimento ou do agravo em saúde. Portanto, há possibilidade de intervenção por meio do psicodrama como gerador do protagonismo dos usuários (KONOPIK e CHEUNG, 2013).

Dessa maneira, é importante apontar que no estudo realizado no Irã, os autores destacaram que a terapia com teatro nos serviços de saúde é uma arte em espaço aberto, para dar voz ao usuário e entender seu processo de adoecimento e sofrimento psíquico. Eles argumentam que, quando essa terapia é realizada com espontaneidade e aceitando improvisos, ela é um mecanismo primordial para o indivíduo demostrar a sua emoção e ser o autor-chave (protagonista) do fenômeno a ser apresentado por intermédio do teatro como forma de terapêutica (LORY, et. al 2012).

Nessa perspectiva, uma outra forma para despertar o protagonismo do usuário, foi por meio da prática do teatro do oprimido em ações no CAPS, por meio da construção de teoria da educação popular nesse cenário. Foi observado que essa prática promoveu a saúde, estimulou a autonomia e o protagonismo social dos usuários, que assumiram um lugar de criação nesse contexto entre a saúde e o sofrimento psíquico (ANDRADE et. al, 2018).

De acordo com Portugal et. al (2018) uma clínica de cuidados que coloca o papel da arte como um mecanismo terapêutico requer um espaço aberto ao protagonismo dos usuários, em um contexto de uma reforma psiquiátrica enquanto processo social complexo e contínuo ${ }^{(16)}$. Para tanto, tais condutas terapêuticas necessitam de um aperfeiçoamento do processo de cuidar no que diz respeito a expressão subjetiva do sofrimento psíquico.

Cabe ressaltar que, segundo Gonzalez et. al. (2018) o psicodrama é um dispositivo terapêutico que proporciona diminuição de sintomas de sofrimento e expressão dos problemas da vida, além de ser uma técnica que permite à pessoa assistida ter espontaneidade na expressão de suas ideias e angustias.

É evidente que o psicodrama e o teatro terapia são manifestações da arte relacionados a uma estética que valoriza a subjetividade e a voz da pessoa assistida. Para tanto, são reconhecidos por meio de uma terapêutica que tende aperfeiçoar a assistência em saúde mental centralizada no indivíduo. Além disso, tanto o psicodrama quanto a terapia com teatro despertam o protagonismo do usuário e a amenização do seu sofrimento.

\section{Limitações do estudo}

Este estudo apresenta algumas limitações a saber: para a obtenção de informações referentes à temática foram utilizadas as bases de dados BVS (BIREME), CINAHL, SCOPUS e PubMed. Entretanto, outras bases de dados nacionais e internacionais poderiam ser 
investigadas, o que pode ter levado, de certo modo, a não inclusão de alguns estudos também importantes.

\section{Contribuições para saúde mental e humanização da assistência}

Este estudo poderá contribuir para o desenvolvimento de estudos futuros sobre a temática do protagonismo do usuário nos serviços de saúde mental, e, igualmente, para o avanço em outras discussões. Além disso, poderá estimular os profissionais e trabalhadores em saúde a pensar e adotar inovações na prática assistencial e a reconhecer as ferramentas das ações possíveis para promover o protagonismo do usuário na assistência em saúde mental, presentes na literatura global. Para tanto, reflete na possibilidade de um aprimoramento dos serviços de saúde para a adotar medidas para a promoção do protagonismo da pessoa na assistência em saúde mental e valorização da autonomia.

\section{Conclusão}

Este estudo analisou as formas como o protagonismo do usuário dos serviços de saúde mental são descritos na literatura global. Na área da saúde mental, o fenômeno é contemporâneo e reverbera uma nova perspectiva da terapêutica descrita nas diretrizes operacionais em saúde mental em diversos países. A finalidade é produzir no dia a dia do usuário um novo olhar sobre si, tornando-o protagonista da sua vida e tendo um processo de cuidado que valorize sua voz e sua subjetividade.

As ações e práticas de cuidado em saúde são descritas como uma oportunidade de alcance à autonomia do usuário, inclusão social e o suporte emocional nos processos da própria vida. Essa iniciativa tende a lograr êxito quando o usuário é colocado no centro da atenção e cuidado. Ela deve acontecer de maneira plural e singular e ser direcionada para o usuário e no contexto onde ele se encontra inserido, não apenas um olhar clínico sobre para a doença ou sofrimento.

O modelo da teoria da Maré, a construção de Projetos Terapêuticos Singulares, a aplicação do Psicodrama e a realização de oficinas terapêuticas, apresentam-se como ferramentas promotoras do protagonismo do usuário nos serviços de saúde mental. Essas práticas são apontadas na literatura mundial como contribuintes à transformação da assistência, tornando-a mais humanitária, reconhecedora das necessidades do usuário e produtora de saúde.

Em suma, o protagonismo do usuário no serviço de saúde mental é um fenômeno que acompanha a reforma psiquiátrica mundial. A literatura global apresenta reflexões sobre o tema 
e elucida alguns dispositivos terapêuticos que permite que o usuário possa ter uma valorização de sua subjetividade e exercer seu protagonismo no espaço clínico. Contudo, há necessidade de formação profissional para adotar tais condutas e espaços de discussão política, a fim de que o usuário possa exercer sua participação social tanto na clínica quanto para além dela.

A reabilitação psicossocial deve preconizar espaços de trocas e não de controle social sobre as pessoas em crise. Portanto, as condutas e os dispositivos terapêuticos que valorizem a subjetividade do usuário, e que permitam que ele se expresse e tenha voz, possibilitarão uma atenção focalizada no protagonismo do usuário e uma racionalidade profissional galgada na dimensão da tríade da clínica- política-ética, como meta de promover a saúde mental.

Por fim, o panorama da literatura global demonstra que há distintas maneiras para se trabalhar o protagonismo do usuário, uma vez que é um fenômeno que ganhou maior atenção, com a reforma psiquiátrica em diversos países.

\title{
PROTAGONISMO DEL PACIENTE EN LA ATENCIÓN DE SALUD MENTAL: UNA REVISIÓN INTEGRATIVA
}

\section{Resumén}

Objetivo: analizar en la literatura científica global el tema del protagonismo del usuario en el servicio de salud mental. Método: revisión bibliográfica integradora, basada en artículos en portugués, inglés y francés en las bases de datos de la Biblioteca Virtual en Salud, CINAHL, SCOPUS y PubMed con palabras clave y MESH, sin determinación temporal. El diagrama de flujo de selección eligió 21 artículos de agosto de 2017 a abril de 2020. Resultados: el protagonismo en el área de salud mental se describe como una práctica transformadora y humanitaria basada en las necesidades del usuario, los artículos nacionales e internacionales aportaron conceptos y acciones sobre protagonismo. Conclusión: el Modelo Maré, los talleres terapéuticos, el Proyecto Terapéutico Singular, el Psicodrama y el teatro son herramientas esenciales para el papel del usuario en la atención de la salud mental, ya que delimitan tecnologías y dispositivos de producción que impregnan la autonomía. La valoración y subjetividad de la persona asistida.

Palabras clave: Protagonismo, Asistencia en Salud Mental, Autonomía personal.

\section{PATIENT LEADERSHIP IN MENTAL HEALTH CARE: AN INTEGRATIVE REVIEW}

\begin{abstract}
Objective: to analyze in the global scientific literature the theme of user leadership in the mental health service. Method: integrative literature review, based on articles in Portuguese, English and French in the databases of the Virtual Health Library, CINAHL, SCOPUS and PubMed with keywords and MESH, without temporal determination. 21 articles were chosen by the selection flowchart from August 2017 to April 2020. Results: the leadership in the mental health area is described as a transformative and humanitarian practice based on the needs of the user, national and international articles brought concepts and actions on leadership. Conclusion: the Barbarói, Santa Cruz do Sul, n. 58, p.<95-121>, jan./jun. 2021
\end{abstract}


Tidal Model, the therapeutic workshops, the Singular Therapeutic Project, Psychodrama and the theater are tools that are essential for the role of the user in mental health care, as they demarcate technologies and production devices that permeate autonomy, the valuation and subjectivity of the assisted person.

Keywords: Leadership, Mental Health Assistance, Personal Autonomy.

\section{REFERÊNCIAS}

ALVES, T.C.; OLIVEIRA, W.F.; VASCONCELOS, E.M. A visão de usuários, familiares e profissionais acerca do empoderamento em saúde mental. Physis: Revista de Saúde Coletiva, Rio de Janeiro, n.23, p.51-71, mar. 2013.Disponível em:

<https://www.scielosp.org/pdf/physis/2013.v23n1/51-71/pt>. Acesso em: 20 mar. 2020.

ANDRADE, L.A.A.; VELOSO, T.M.G. Arte e saúde mental: uma experiência com a metodologia participativa da Educação Popular. Pesqui. prát. Psicossociais, São João del-Rei, v.10, n.1, p.79-87, jan./jun. 2015.Disponível em:

<http://pepsic.bvsalud.org/pdf/ppp/v10n1/07.pdf>. Acesso em: 07 mar. 2020.

BARKER, P.; BARKER P. B. The Tidal Model of Mental Health Recovery and Reclamation: Application in Acute Care Settings. Issues Ment Health Nurs, Londres, v.31 n.3, p.171-80, mar. 2010. Disponível em:

<https://www.tandfonline.com/doi/full/10.3109/01612840903276696>. Acesso em: 18 mar. 2018 .

COELHO, R.S.; VELÔSO, T.M.G.; BARROS, S.M.M. Oficinas com Usuários de Saúde Mental: a Família como Tema de Reflexão. Psicologia: Ciência e Profissão, [s.1.], v.37, n.2, p.489-99, abr./jun. 2017. Disponível em:<http://www.scielo.br/pdf/pcp/v37n2/1982-3703pcp-37-2-0489.pdf. > Acesso em: 08 mar. 2020.

COSTA, D.F.C.; PAULON, S.M. Participação Social e protagonismo em saúde mental: a insurgência de um coletivo Social. Saúde debate, Rio de Janeiro, v.36, n.95, p.575-82, out./dez. 2012.Disponível em:< http://www.scielo.br/pdf/sdeb/v36n95/a09v36n95.pdf.> Acesso em: 14 mar. 2020.

DUTRA, V.F.D.; BOSSATO, H.R.; OLIVEIRA, R.M.P. Mediar a autonomia: um cuidado essencial em saúde mental. Esc. Anna Nery, Rio de Janeiro, v.21, n.3, fev. 2017. Disponível em:<http://www.scielo.br/pdf/ean/v21n3/pt_1414-8145-ean-2177-9465-EAN-2016-0284.pdf. $>$ Acesso em: 10 mar. 2020.

FABRI, J.M.G.; LOYOLA, C.M.L. Desafios e necessidades atuais da enfermagem psiquiátrica. Rev enferm UFPE on line., Recife, v.8, n.03, p.695-701, mar. 2014. Disponível em:< https://periodicos.ufpe.br/revistas/revistaenfermagem/article/view/9727/9817>. Acesso em: 07 mar. 2020.

FARIAS, I.D. et al. Oficina Terapêutica como expressão da subjetividade. Rev. Eletrônica Saúde Mental Álcool e Drog., [s.1.], v.16, n.3, p.147-153, jul./set. 2016.Disponível em: < https://www.redalyc.org/pdf/803/80347135003.pdf.> Acesso em: 15 jan. 2020.

FREITAS, F.; AMARANTE, P. Medicalização em psiquiatria. Rio de Janeiro: Editora FIOCRUZ., 2017. 
GONZALEZ, A.J.; MARTINS P.; LIMA M.P. Studying the efficacy of psychodrama with the Hermeneutic Single Case Efficacy Design: Results from a longitudinal study. Frontiers in Psychology, [s.1.], v. 9, n.1662, set. 2018. Disponível em:

<https://www.ncbi.nlm.nih.gov/pmc/articles/PMC6139396/pdf/fpsyg-09-01662.pdf> Acesso em: 10 mar. 2020.

GRUSKA, V.; DIMENSTEN, M. Reabilitação Psicossocial e Acompanhamento Terapêutico: equacionando a reinserção em saúde mental. Psicologia Clínica, [s.1.], v.27, n.1, p.101-122, set. 2015. Disponível em: < http://www.scielo.br/pdf/pc/v27n1/0103-5665-pc-27-0100101.pdf > Acesso em: 20 mar. 2020.

JORGE, M.S.B. et al. Apoio matricial, projeto terapêutico singular e produção do cuidado em saúde mental. Texto contexto - enferm., Florianópolis, v.24, n.1, p.112-120, jan./mar. 2015. Disponível:< http://www.scielo.br/pdf/tce/v24n1/0104-0707-tce-24-01-00112.pdf>. Acesso em: 20 mar. 2020.

JUNQUEIRA, A.M.G.; CARNIEL, I.C.; MANTOVANI, A. As assembleias como possibilidades de cuidado em saúde mental em um CAPS. Vínculo- Revista do NESME, São Paulo, v.12, n.1, p.31-40, 2015.Disponível em:<http://pepsic.bvsalud.org/pdf/vinculo/v12n1/v12n1a06.pdf >.Acesso em: 10 mar. 2020.

KANTORSKI, L.P. et al. Grupos de ouvidores de vozes: estratégias e enfrentamentos. Saúde debate, Rio de Janeiro, v.4, n.115, p. 1143-55, out./dez. 2017. Disponível em:<http://www.scielo.br/pdf/sdeb/v41n115/0103-1104-sdeb-41-115-1143.pdf > Acesso em : 10 mar. 2020.

KIDD, Jacquie. Cultural boundary surfing in mental health nursing: a creative narration. Contemporary Nurse, Auckland, v.34, n.2, p.277-88, out. 2010. Disponível em: $<$ https://www.researchgate.net/publication/44637333_Cultural_boundary_surfing_in_mental_ health_nursing_A_creative_narration>. Acesso em: 07 mar. 2020.

KONOPIK, A.D.; CHEUNG, M. Psychodrama as a Social Work Modality. Soc. Work, [s.1],v.58, n.1, p.9-20, set. 2013. Disponível em: $<$ https://www.researchgate.net/publication/235626655_Psychodrama_as_a_Social_Work_Mo dality>. Acesso em: 20 mar. 2020.

LORY, S.S. et al. Theater therapy and its integration with improvisation. Life Science Journal,[s.1],v.9, n.4, p.254-266, maio 2012. Disponível em: <http://www.lifesciencesite.com/lsj/life0904/592_13092life0904_3971_3978.pdf>. Acesso em: 10 mar. 2020.

MALTA, D.C. et al. A implementação das prioridades da Política Nacional de Promoção da Saúde, um balanço, 2006 a 2014. Ciênc. saúde coletiva, v.19, n.11, p.4301-12, nov. 2014. Disponível em: <http://www.scielo.br/pdf/csc/v19n11/1413-8123-csc-19-114301.pdf>.Acesso em: 20 mar. 2020.

MELNYK, B.M.; FINEOUT-OVERHOLT, E. In: Evidence-based practice in nursing \& healthcare: a guide to best practice. Philadelphia: Lippincot Williams \& Wilkins, 2005. p.3-24. 
MENDES, K.D.S.; SILVEIRA, R.C.C.P.; GALVÃO, C.M. Revisão integrativa: método de pesquisa para a incorporação de evidências na saúde e na enfermagem. Texto contexto Enferm., Florianópolis, v.17, n.4, p.758-64, out. 2008. Disponível em: <http://www.scielo.br/pdf/tce/v17n4/18.pdf>. Acesso em: 08 mar. 2020.

MINISTÉRIO DA SAÚDE. Secretaria de Atenção à Saúde. Departamento de Atenção Básica. Núcleo de Apoio à Saúde da Família. Ferramentas para a gestão e para o trabalho cotidiano. Cadernos de Atenção Básica, n. 39. Brasília: MS, 2014.

PACHECO, S.U.C.; RODRIGUES, S.R.; BENATTO, M.C. A importância do empoderamento do usuário de CAPS para a (re)construção do seu projeto de vida. MentalBarbacena, v.12, n.22, p.72-89, jan./jun. 2018. Disponível em: <http://pepsic.bvsalud.org/pdf/mental/v12n22/v12n22a06.pdf>. Acesso em: 12 mar. 2020.

PASSOS, E. et al. Autonomia e cogestão na prática em saúde mental: o dispositivo da gestão autônoma da medicação (GAM). Aletheia, Canoas, n.41, p.24-38, maio/ago. 2013. Disponível em:< http://pepsic.bvsalud.org/pdf/aletheia/n41/n41a03.pdf > Acesso em: 10 mar. 2020.

PINHO, E.S.; SOUZA, A.C.S.; ESPERIDIAO, E. Processos de trabalho dos profissionais dos Centros de Atenção Psicossocial: revisão integrativa. Ciênc. saúde coletiva,[s.1], v.23,n.1, p.141-152, 2018. Disponível em: < http://www.scielo.br/pdf/csc/v23n1/en_1413-8123-csc-2301-0141.pdf>. Acesso em:10 mar. 2020.

PITTA, A.M. F.; MACEDO, D.C.; ROCHA, C.C.M. Direitos humanos nos Centros de Atenção Psicossocial do Nordeste do Brasil: um estudo avaliativo, tendo como referência o Quality Rights - WHO. Saúde debate, Rio de Janeiro, v.39, n.106, p.760-71, jul./ago. 2015.Disponível em: < http://www.scielo.br/pdf/sdeb/v39n106/0103-1104-sdeb-39-10600760.pdf >.Acesso em: 07 mar. 2020.

PORTUGAL, C.M.; MEZZA, M.; NUNES, M. A clínica entre parênteses: reflexões sobre o papel da arte e da militância na vida de usuários de saúde mental. Physis, Rio de Janeiro,v.28, n.2, ago. 2018. Disponível em: < http://www.scielo.br/pdf/physis/v28n2/0103-7331-physis28-02-e280211.pdf>. Acesso em: 12 mar. 2020.

RIBEIRO, M.C.; BEZERRA, W.C. A reabilitação psicossocial como estratégia de cuidado: percepções e práticas desenvolvidas por trabalhadores de um serviço de saúde mental. Rev Ter Ocup Univ, São Paulo, v.26, n.3, p.301-08, set./dez. 2015.Disponível em: <file:///C:/Users/hercu/Downloads/89628-Texto\%20do\%20artigo-199973-1-10-20160212.pdf $>$.Acesso em: 20 mar. 2020.

RUELLAND, I. Les dispositifs de délibération en santé mentale et la démocratisation des échanges entre les pairs: le cas de l'assemblée des usagers d'un centre d'attention psychosociale au Brésil. Santé mentale au Québec, Quebec, v.40, n.1, p.153-170, jan. 2015. Disponível em: <https://www.erudit.org/fr/revues/smq/2015-v40-n1smq02004/1032388ar.pdf >. Acesso em: 10 jan. 2020.

SANTIAGO, E.; SANTOS, L. Uma etnografia da atenção à saúde mental de sujeitos em situação de rua. Barbarói, Santa Cruz do Sul, n.55, p 81- 101, jul./dez. 2019. Disponível em: <http://dx.doi.org/10.17058/barbaroi.v0i0.13973 >. Acesso em: 15 jan.2020. 
SANTOS, P.L.T.; BITENCOURT, L.C. Psicodrama, ética e o outro. Rev. Bra.Psicodrama, São Paulo, v.27, n.2, p.174-85, 2019. Disponível em:

<http://revbraspsicodrama.org.br/rbp/article/view/7/3 >. Acesso em: 15 jan. 2020.

SILVA, T.A.; PAULA JUNIOR, J.D.; ARAUJO, R.C. Centro de Atenção Psicossocial (CAPS): ações desenvolvidas em município de Minas Gerais, Brasil. Rev. latinoam. psicopatol. fundam, São Paulo, v.21, n.2, p.346-363, jun. 2018.Disponível em: <http://www.scielo.br/pdf/rlpf/v21n2/1415-4714-rlpf-21-2-0346.pdf >. Acesso em: 12 mar.2020.

STETLER, Cheryl B. Updating the Stetler Model of research utilization to facilitate evidencebased practice. Nurs Outlook, [s.1.] n.49, p.272-9, nov./dez. 2001.Disponível em: <https://www.nursingoutlook.org/article/S0029-6554(01)47839-0/fulltext >. Acesso em: 20 jan. 2020.

SUMSION, T.; LENCUCHA R. Balancing challenges and facilitating factors when implementing client-centered collaboration in a mental health setting. British Journal of Occupational Therapy,[s.1], v.70, n.12,p.513-20, nov. 2007. Disponível em: https://www.researchgate.net/profile/Raphael_Lencucha/publication/233610580_Balancing_ Challenges_and_Facilitating_Factors_when_Implementing_ClientCentred_Collaboration_in_a_Mental_Health_Setting/links/57df21db08ae5292a37f498c/Balan cing-Challenges-and-Facilitating-Factors-when-Implementing-Client-Centred-Collaborationin-a-Mental-Health-Setting.pdf. Acesso em: 18 mar. 2020.

TONG, A.; SAINSBURY, P.; CRAIG J. Consolidated criteria for reporting qualitative research (COREQ): a 32-item checklist for interviews and focus groups. Int J Qual Health Care, [s.1], v.19, n.6, p.349-57, set.2007.Disponível em:

<https://academic.oup.com/intqhe/article/19/6/349/1791966 >.Acesso em: 20 mar. 2020. TORRACO, Richard J. Writing Integrative Literature Reviews: Using the Past and Present to Explore the Future. Hum Resour Dev Rev,[s.1], v.15, n.4,p.404-428, out. 2016.Disponível em: <https://journals.sagepub.com/doi/abs/10.1177/1534484316671606 >. Acesso em: 15 dez. 2019.

TORRE, E.H.G.; AMARANTE, P. Protagonismo e subjetividade: a construção coletiva no campo da saúde mental. Ciênc. saúde coletiva, [s.1], v.6, n.1, p. 73-85, 2001. Disponível em: http://www.scielo.br/pdf/csc/v6n1/7026.pdf Acesso em 07 dez 2018.

VASCONCELOS, M.G.F. et al. Projeto terapêutico em Saúde Mental: práticas e processos nas dimensões constituintes da atenção psicossocial. Interface, Botucatu, v.20, n.57, p.313-23, 2016. Disponível em: < http://www.scielo.br/pdf/icse/v20n57/1807-5762-icse-20-570313.pdf>. Acesso em:15 jan. 2020.

Data de recebimento: $12 / 05 / 2020$

Data de aceite: 03/01/2021

\section{Sobre os autores:}

Hércules Rigoni Bossato é Enfermeiro, Doutor em Enfermagem pela Universidade Federal do Barbarói, Santa Cruz do Sul, n. 58, p.<95-121>, jan./jun. 2021 
Rio de Janeiro/UFRJ, Mestre em Ciências do Cuidado em Saúde pela Universidade Federal Fluminense/UFF, Especialista em Saúde da Família Multiprofissional pela Universidade Federal Fluminense. Bacharel e Licenciado em Enfermagem pela Universidade Federal Fluminense. Atualmente é professor adjunto da Universidade Federal do Rio de Janeiro/Campus Macaé. Tem experiência em ensino e pesquisa na área de Enfermagem, com ênfase em: Reabilitação físico-motora; Reabilitação Psicossocial; Saúde Coletiva; Saúde Mental; Estratégia de Saúde da Família; Políticas Públicas de Saúde; Gestão em Saúde e Educação em Saúde. Endereço Eletrônico: herculesbossato@gmail.com

Virginia Faria Damasio Dutra é Doutora em Enfermagem pela Escola de Enfermagem Anna Nery da Universidade Federal do Rio de Janeiro/UFRJ (2015), Mestre em Enfermagem pela Universidade do Estado do Rio de Janeiro/UERJ (2006), Especialista em Atenção Psicossocial pela UFRJ (2003), Graduada em Enfermagem pela Universidade Federal de Goiás/UFG (2000). Professora da Escola de Enfermagem Ana Nery/UFRJ Departamento Médico-Cirúrgica na área de Saúde Mental. Chefia de Enfermagem do Instituto de Psiquiatria/UFRJ (2018) Tem experiência na área de Enfermagem, com ênfase em Enfermagem Psiquiátrica, atuando principalmente nos seguintes temas: enfermagem, saúde mental, ensino e cuidado. Endereço Eletrônico: virginia.damasio@gmail.com

Albert Lengruber de Azevedo é Doutor e Mestre em Enfermagem pela Escola de Enfermagem Anna Nery, da Universidade Federal do Rio de Janeiro (EEAN/UFRJ). Professor do Centro Universitário Belford Roxo e da Faculdade de Duque de Caxias, RJ - Brasil. Endereço Eletrônico: albertenfermagem@yahoo.com.br

Paula Cristina da Silva Cavalcanti é Enfermeira. Doutora em Enfermagem pela Escola de Enfermagem Anna Nery da Universidade Federal do Rio de Janeiro-UFRJ. Professora da Associação Educacional Dom Bosco- RJ. Endereço Eletrônico: pcscavalcanti@gmail.com

Cristina Maria Douat Loyola é Enfermeira. Doutora em Saúde Coletiva pelo Instituto de Medicina Social- IMS da Universidade Estadual do Rio de Janeiro- UERJ. Professora do Mestrado em Gestão de Programas e Serviços de Saúde da Universidade CEUMA, São Luís MA. Endereço Eletrônico: crisloyola@hotmail.com

Rosane Mara Pontes de Oliveira é Enfermeira, Doutora de Enfermagem pela Escola de Enfermagem Anna Nery- UFRJ. Professora Associada da Universidade Federal do Rio de Janeiro no Departamento de Enfermagem Médico-Cirúrgica. Endereço Eletrônico: romapope@gmail.com 